\title{
Atividade antimicrobiana de extratos hidroalcólicos de espécies da coleção de plantas medicinais CPQBA/UNICAMP
}

Duarte, M.C.T. ;Figueira, G.M.* ; Pereira, B. ; Magalhães, P.M. Delarmelina, $\mathbf{C}$.

${ }^{1}$ Centro Pluridisciplinar de Pesquisas Químicas, Biológicas e Agrícolas (CPQBA/UNICAMP)

\section{Resumo}

Extratos obtidos a partir de 45 espécies da Coleção de Germoplasmas do CPQBA foram estudados quanto à atividade antimicrobiana. As espécies que apresentaram forte inibição (Concentração Mínima Inibitória até $0,5 \mathrm{mg} / \mathrm{mL}$ ) para os respectivos microrganismos foram: Achillea millefolium (0,5), Mikania laevigata $(0,04)$, Solidago chilensis $(0,1)$, Piper marginatum $(0,2)$ para Staphylococcus aureus; Aloysia gratissima (0,1), P. marginatum (0,2), M. laevigata $(0,09)$ para Bacillus subtilis e Mentha pullegium $(0,3)$, Mikania glomerata $(0,1)$, M. laevigata $(0,04)$, Stachytarpeta cayenensis $(0,2)$ e Bacharis dracunculifolia $(0,5)$ para Streptococcus faecium. De acordo com os resultados, ressaltamos a espécie $M$. laevigata por apresentar inibição contra três das bactérias estudadas, em concentrações similares a do cloranfenicol, padrão de referência utilizado.

Abstract

Crude extracts of 45 medicinal plants from CPQBA Germoplasm Collection were tested for their antimicrobial activity. The species that presented strong activity (Minimal Inhibitory Concentration until $0.5 \mathrm{mg} \cdot \mathrm{mL}^{-1}$ ) against the respective microorganisms were: Achillea millefolium (0.5), Mikania laevigata (0.04), Solidago chilensis (0.1), Piper marginatum (0.2) against Staphylococcus aureus; Aloysia gratissima (0.1), P. marginatum (0.2), M. laevigata (0.09) against Bacillus subtilis and Mentha pullegium (0.3), Mikania glomerata (0.1), M. laevigata (0.04), Stachytarpeta cayenensis (0.2) and Bacharis dracunculifolia (0.5) against Streptococcus faecium. According to results, M. laevigata was able to inhibit three bacteria species, at similar concentrations as observed for the standard chloranphenicol.

A atividade biológica de plantas medicinais tem sido objeto de intensa investigação científica. Plantas superiores e aromáticas são amplamente utilizadas na medicina popular, uma vez que apresentam amplo espectro de atividade e inibição comprovada contra bactérias e fungos ${ }^{1}$. A maioria dessas propriedades é conferida por produtos do 
metabolismo secundário, como terpenóides e compostos fenólicos, que também na forma pura exibem atividade ${ }^{2}$.

Extratos e óleos de várias espécies mostraram-se eficientes no controle de fungos relacionados a infecções da pele ${ }^{2}$, sobre bactérias patogênicas bucais ${ }^{3}$, e sobre uma variedade de bactérias Gram-negativas e Gram-positivas ${ }^{4}$.

Trabalhos recentes sobre a atividade antimicrobiana de extratos e óleos essenciais mostram o grande potencial de aplicação de plantas nativas de diversas regiões do mundo. No Brasil, estudos com a mesma finalidade são de grande importância, uma vez que plantas medicinais são utilizadas em várias áreas da saúde como forma alternativa de tratamento. Além disso, nosso país apresenta uma rica biodiversidade, devendo-se considerar o custo mais baixo destas formas terapêuticas em relação a medicamentos industrializados.

O objetivo deste trabalho foi estudar a atividade antimicrobiana de extratos de 45 plantas medicinais pertencentes à Coleção de Germoplasmas do CPQBA/ UNICAMP

Tabela 1. Plantas medicinais da Coleção de Germoplasmas do CPQBA/UNICAMP com atividade antimicrobiana e respectivos microrganismos.

\begin{tabular}{|c|c|c|c|c|}
\hline Planta Medicinal & Número Herbário & $\begin{array}{c}\text { Rendimento } \\
\text { Extrato }(\% \mathbf{p} / \mathbf{p})\end{array}$ & Microrganismo & MIC mg.mL-1 \\
\hline Achyrocline satureoides (DC.)Lam. & UEC 127.116 & 32,7 & B. subtilis & 1,6 \\
\hline Achillea millefolium $\mathrm{L}$. & UEC 127.114 & 61,6 & S. aureus & 0,5 \\
\hline A. millefolium $\mathrm{L}$. & UEC 127.114 & 61,6 & B. subtilis & 1,6 \\
\hline Aloysia gratissima (Gill \& Hook) & UEC 121.393 & 20,8 & B. subtilis & 0,1 \\
\hline Artemisia annua L. & CPQBA1246 & 34,5 & B. subtilis & 1,7 \\
\hline Baccharis dracunculifolia DC. & CPQBA 622 & 46,4 & S. aureus & 1,6 \\
\hline B. dracunculifolia DC. & CPQBA 622 & 46,4 & S. faecium & 0,5 \\
\hline Cordia curassavica (Jacq.) Roem. & UEC112744 & 15,8 & B. subtilis & 0,6 \\
\hline Hydrocotile asiatica $\mathrm{L}$. & UEC 127.111 & 36,3 & B. subtilis & 1,5 \\
\hline Mentha pulegium $\mathrm{L}$. & UEC 121.402 & 43,6 & S. faecium & 0,3 \\
\hline Mikania glomerata Sprengel. & UEC 102047 & 18,0 & S. faecium & 0,1 \\
\hline M. laevigata Sch. Bip. ex Baker & UEC 102044 & 30,0 & S. aureus & 0,04 \\
\hline M. laevigata Sch. Bip. ex Baker & UEC 102044 & 30,0 & S. faecium & 0,35 \\
\hline M. laevigata Sch. Bip. ex Baker & UEC 102044 & 30,0 & B. subtilis & 0,09 \\
\hline Piper aduncum L. & UEC 127.118 & 32,63 & S. aureus & 1,8 \\
\hline P. aduncum $\mathrm{L}$. & UEC 127.118 & 32,63 & B. subtilis & 0,9 \\
\hline P. marginatum Jacq. & UEC 121.395 & 27,3 & S. aureus & 0,2 \\
\hline P. marginatum Jacq. & UEC 121.395 & 27,3 & B. subtilis & 0,2 \\
\hline Solidago chilensis Meyen & UEC 121.391 & 35,7 & S. aureus & 0,1 \\
\hline S. chilensis Meyen & UEC 121.391 & 35,7 & S. faecium & 1,6 \\
\hline Stachys byzantina C. Koch. & UEC 121.404 & 46,8 & B. subtilis & 1,9 \\
\hline Stachytarpeta cayenensis L.C. & UEC 121.394 & 40,3 & S. faecium & 0,2 \\
\hline Vetiveria zizanoides Stapf. & UEC 121.415 & 5,77 & S. aureus & 1,1 \\
\hline V. zizanoides Stapf. & UEC 121.415 & 5,77 & B. subtilis & 0,6 \\
\hline
\end{tabular}

\section{Material e Método}

Após secagem das plantas em estufa à $40^{\circ} \mathrm{C}$ durante $48 \mathrm{~h}$ e determinação do teor de umidade, os extratos foram obtidos a partir de $10 \mathrm{~g}$ das plantas moídas com solução hidroalcoólica (70:30), seguido de agitação a $200 \mathrm{rpm}$ em temperatura ambiente, por $3 \mathrm{~h}$. Os extratos foram filtrados e os resíduos re-extraídos. Finalmente, os filtrados combinados foram evaporados a vácuo até a secura.

A atividade antimicrobiana foi determinada através do teste da microdiluição ${ }^{5}$. Os extratos, diluídos em água e Tween 80, foram testados a concentrações entre 2,0 - 0,031 mg. $\mathrm{ml}^{-1}$ contra os seguintes microrganismos: Bacillus subtilis CCT 2576, Escherichia coli CCT 0547, Staphylococcus aureus CCT 2740, Staphylococcus epidermides ATCC 12228, Micrococcus luteus CCT 2692, Rhodococcus equi CCT 0541, Salmonella choterasuis CCT 4296, Pseudomonas aeruginosa ATCC 13388, Enterococcus faecium CCT 5079, Streptococcus faecium ATCC 10541 e Candida albicans ATCC 10231. Os inóculos foram preparados a partir de culturas de crescimento recente (24 h), cuja turvação foi comparada à escala McFarland $(0,5)$ e 
diluídos para concentração final de $10^{4}$ células.m ${ }^{-1}$. Após incubação dos microrganismos na presença dos extratos por $24 \mathrm{~h}$ a $37^{\circ} \mathrm{C}, 50 \mu \mathrm{L}$ de solução $0,5 \%$ de TTC (cloreto de trifenil tetrazolium) foram adicionados e a placa re-incubada por 3 h. A MIC (Concentração Mínima Inibitória) foi definida como a menor concentração do extrato capaz de impedir o aparecimento de coloração vermelha. Como padrão de referência foi utilizado cloranfenicol (bactérias) ou nistatina (Candida albicans).

A Tabela 1 apresenta os resultados de rendimento e Concentração Mínima Inibitória (MIC) dos extratos com atividade, dentro da faixa de concentração estudada. Segundo Aligiannis et al. ${ }^{6}$ podemos considerar como de forte atividade, compostos obtidos a partir de plantas medicinais e aromáticas que apresentem MIC até $0,5 \mathrm{mg} / \mathrm{ml}$, conforme observado para Achillea millefolium, Mikania laevigata, Solidago chilensis, Piper marginatum contra Staphylococcus aureus; Aloysia gratissima, P. marginatum, M. laevigata contra Bacillus subtilis e Mentha pullegium, Mikania glomerata, M. laevigata, Stachytarpeta cayenensis e Bacharis dracunculifolia contra Streptococcus faecium.

M. laevigata destacou-se por apresentar inibição contra três das bactérias estudadas, em concentrações similares as do cloranfenicol. Tal atividade provavelmente esteja relacionada com a presença de compostos da classe dos ácidos diterpênicos, ácidos caurenóico e cupressênico, principais constituintes dos extratos de $M$. laevigata, que quando testados isoladamente (dados não publicados) apresentaram forte atividade antimicrobiana.

\section{Referências}

${ }^{1}$ Hulin, V.; Mathot, A. G.; Mafart, P.; Dufossé, L. - Les proprietés anti-microbiennes des huiles essentielles et composés d'arômes. Sciences des Aliments v.18 p.563-582, 1998.

${ }^{2}$ Adam, K.; Sivropoulou, A.; Kokkini, S.; Lanaras, T.; Arsenakis, M. - Antifungal activities of Origanum vulgare subsp. hirtum, Mentha spicata, Lavandula angustifolia, and Salvia fruticosa Essential Oils against Human Pathogenic Fungi. J. Agric. Food Chem. V.46 p. 17391745, 1998.

${ }^{3}$ Cecanho, R.; Koo, H.; Rosalen, P. L. J. A.; Park, Y. K.; Cury, J. A. - Efeito do extrato hidroetanólico de Mikania laevigata sobre o crescimento bacteriano e a produção de glucamos por estreptococcus do grupo mutans. Anais da XIV Reunião Anual da FESBE, Caxambu - MG, v14 p290 (resumo \# 12.095), 1999.

${ }^{4}$ Galli, A.; Franzetti, L.; Briguglio, D. - Attività antimicrobica in vitro di oli essenziali ed estratti di spezie di uso alimentare. Industrie Alimentari, p.463-466, 1985.

${ }^{5}$ Ellof, J.N. A sensitive and quick microplate method to determine the minimal inhibitory concentration of plant extracts for bacteria. Planta Medica v. 64 n.8 p.711-713, 1998.

${ }^{6}$ Aligiannis N., Kalpotzakis E., Mitaku S., Chinou I.B. Composition and antimicrobial activity of the essential oils of two Origanum species. J. Agric. Food Chem. v.40 n.4168-4170, 2001.

\section{*Autora para correspondência}

Glyn Mara Figueira

Divisão de Agrotecnologia - CPQBA/UNICAMP

Caixa Postal 6171, CEP13081-970, Campinas, SP

e-mail: glyn@cpqba.unicamp.br 